\title{
The Tilings of Deficient Squares by Ribbon $L$-Tetrominoes Are Diagonally Cracked
}

\author{
Viorel Nitica \\ Department of Mathematics, West Chester University, West Chester, PA, USA \\ Email: vnitica@wcupa.edu
}

How to cite this paper: Nitica, V. (2017) The Tilings of Deficient Squares by Ribbon $L$-Tetrominoes Are Diagonally Cracked. Open Journal of Discrete Mathematics, 7, 165-176.

https://doi.org/10.4236/ojdm.2017.73015

Received: May 6, 2017

Accepted: July 9, 2017

Published: July 12, 2017

Copyright (c) 2017 by author and Scientific Research Publishing Inc. This work is licensed under the Creative Commons Attribution International License (CC BY 4.0).

http://creativecommons.org/licenses/by/4.0/ (c) (i) Open Access

\section{Abstract}

We consider tilings of deficient rectangles by the set $T_{4}$ of ribbon $L$-tetrominoes. A tiling exists if and only if the rectangle is a square of odd side. The missing cell has to be on the main NW-SE diagonal, in an odd position if the square is $(4 m+1) \times(4 m+1)$ and in an even position if the square is $(4 m+3) \times(4 m+3)$. The majority of the tiles in a tiling follow the rectangular pattern, that is, are paired and each pair tiles a $2 \times 4$ rectangle. The tiles in an irregular position together with the missing cell form a NW-SE diagonal crack. The crack is located in a thin region symmetric about the diagonal, made out of a sequence of $3 \times 3$ squares that overlap over one of the corner cells. The crack divides the square in two parts of equal area. The number of tilings of a $(4 m+1) \times(4 m+1)$ deficient square by $T_{4}$ is equal to the number of tilings by dominoes of a $2 m \times 2 m$ square. The number of tilings of a $(4 m+3) \times(4 m+3)$ deficient square by $T_{4}$ is twice the number of tilings by dominoes of a $(2 m+1) \times(2 m+1)$ deficient square, with the missing cell placed on the main diagonal. In both cases the counting is realized by an explicit function which is a bijection in the first case and a double cover in the second. If an extra $2 \times 2$ tile is added to $T_{4}$, we call the new tile set $T_{4}^{+}$. A tiling of a deficient rectangle by $T_{4}^{+}$exists if and only if the rectangle is a square of odd side. The missing cell has to be on the main NW-SE diagonal, in an odd position if the square is $(4 m+1) \times(4 m+1)$ and in an even position if the square is $(4 m+3) \times(4 m+3)$. The majority of the tiles in a tiling follow the rectangular pattern, that is, are either paired tetrominoes and each pair tiles a $2 \times 4$ rectangle, or are $2 \times 2$ squares. The tiles in an irregular position together with the missing cell form a NW-SE diagonal crack. The crack is located in a thin region symmetric about the diagonal, made out of a sequence of $3 \times 3$ squares that overlap over one of the corner cells. The number of tilings of a $(4 m+1) \times(4 m+1)$ deficient square by $T_{4}^{+}$is greater than the 
number of tilings by dominoes and monomers of a $2 m \times 2 m$ square. The number of tilings of a $(4 m+3) \times(4 m+3)$ deficient square by $T_{4}^{+}$is greater than twice the number of tilings by dominoes and monomers of a $(2 m+1) \times(2 m+1)$ deficient square, with the missing cell placed on the main diagonal. We also consider tilings by $T_{4}$ and $T_{4}^{+}$of other significant deficient regions. In particular we show that a deficient first quadrant, a deficient half strip, a deficient strip or a deficient bent strip cannot be tiled by $T_{4}^{+}$. Therefore $T_{4}$ and $T_{4}^{+}$give examples of tile sets that tile deficient rectangles but do not tile any deficient first quadrant, any deficient half strip, any deficient bent strip or any deficient strip.

\section{Keywords}

Tiling, Deficient Rectangles, Ribbon Tetromino

\section{Introduction}

We study tilings of deficient rectangles placed in a square lattice by tile sets consisting of polyominoes. A $1 \times 1$ square in the lattice is called a cell or monomer. We call a rectangle deficient if a cell is missing. Similarly one can define deficient quadrants, deficient half strips, or deficient plane. Our tile sets consist either only of ribbon $L$-tetrominoes $\left(T_{4}\right)$ or of ribbon $L$-tetrominoes and a $2 \times 2$ square $\left(T_{4}^{+}\right)$. See Figure 1. A tiling of a region is a covering without overlappings. Only translations of the tiles are allowed in a tiling. A $2 \times 2$ square is said to be 2 -square if it has the coordinates of all vertices even.

The tile sets $T_{4}$ and $T_{4}^{+}$were introduced in [1] [2], motivated by a problem in recreational mathematics. It is shown in [1] [2] that the tile sets have several remarkable properties. Many of them are consequences of the fact that any tiling of the first quadrant by $T_{4}$ or $T_{4}^{+}$follows the rectangular pattern, that is, the tiling reduces to a tiling by $2 \times 4$ rectangles, in which every rectangle is tiled by two tiles from $T_{4}$, and $2 \times 2$ squares. This in turn, is a consequence of the fact that in any covering without overlaps of a region in the first quadrant bounded by a step 2 staircase and the coordinate axes, the 2 -squares are all covered by $2 \times 4$ rectangles, covered by two tiles from $T_{4}$, and 2 -squares. A bit unexpected, these results have applications to tilings of deficient regions. They also provide a natural mechanism for producing tilings with cracks.

Our main results are the following.
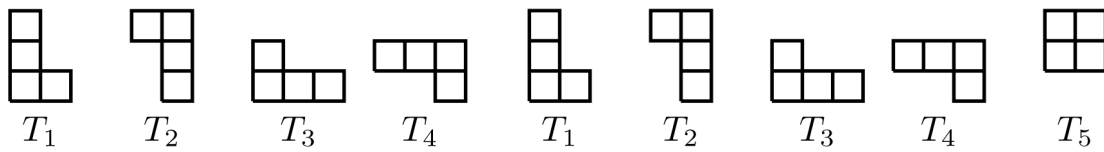

(a)

(b)

Figure 1. Tile sets. (a) The tile set $T_{4}$; (b) The tile set $T_{4}^{+}$. 
Theorem 1. Assume that a deficient rectangle is tiled by $T_{4}$. Then the following are true:

1. The rectangle is a square of odd side $(2 n+1) \times(2 n+1), n \geq 1$.

2. The missing cell is on the main NW-SE diagonal, in an odd position for a $(4 m+1) \times(4 m+1)$ square and in an even position for a $(4 m+3) \times(4 m+3)$ square. For all $m \geq 1$, all such deficient squares can be tiled by $T_{4}$.

3. Most of the tiles (all but $n$ or $n+1$ ) in a tiling follow the rectangular pattern, that is, are paired and each pair tiles a $2 \times 4$ rectangle.

4. (Existence of the crack) The $n$ or $n+1$ tiles in an irregular position together with the missing cell form a NW-SE diagonal crack starting in the upper left corner of the square and ending in the lower right corner.

5. (Location of the crack) The crack is located in a thin region symmetric about the diagonal, made out of a sequence of $3 \times 3$ squares that overlap over one of the corner cells.

6. (Symmetry of the crack) The crack divides the square in two parts of equal area.

7. The number of possible cracks (counted twice if they allow for two different tilings) in a fixed deficient square is equal to $C_{2 m}^{m}$ for a $(4 m+1) \times(4 m+1)$ square and $2 C_{2 m}^{m}$ for a $(4 m+3) \times(4 m+3)$ square.

8. The number of tilings of a $(4 m+1) \times(4 m+1), m \geq 1$, deficient square by $\mathrm{T}_{4}$ is equal to the number of tilings by dominoes of a $2 \mathrm{~m} \times 2 \mathrm{~m}$ square.

9. The number of tilings of a $(4 m+3) \times(4 m+3), m \geq 1$, deficient square by $T_{4}$ is twice the number of tilings by dominoes of a $(2 m+1) \times(2 m+1)$ deficient square, with the missing cell placed on the main diagonal.

10. The counting is realized by a bijection in 8. and a double cover in 9.. They takes a tiling by $T_{4}$ into a tiling by dominoes: for a tiling of the deficient square by $T_{4}$, eliminate the crack, replace the pairs of tiles that cover $2 \times 4$ rectangles by $2 \times 4$ rectangles, reassemble the remaining two region in a square and then do a $1 / 2$-homothety.

11. (Propagation of the crack) The crack and the tiling of a $(2 n+1) \times(2 n+1)$ deficient square can be extended (imbedded) into a crack (tiling) of a $(2 n+5) \times(2 n+5)$ deficient square.

The tilings in Figure 2 illustrate the statement of the theorem. The number of tilings in 8 . is independent of the position of the missing cell on the diagonal and can be computed using Kasteleyn formula [3]. The number of tilings in 9. depends on the position of the missing cell on the diagonal. For example, if $m=2$ the numbers of tilings by $T_{4}$ of a $11 \times 11$ deficient square are, in this order, $384,224,392,224,384$. These are twice the numbers of tilings by dominoes of a $5 \times 5$ deficient square with the missing cell placed on the diagonal.

Theorem 2. Assume that a deficient rectangle is tiled by $T_{4}^{+}$. Then the following are true:

1. The rectangle is a square of odd side $(2 n+1) \times(2 n+1), n \geq 1$.

2. The missing cell is on the main NW-SE diagonal, in an odd position if the square is $(4 m+1) \times(4 m+1)$ and in an even position if the square is 


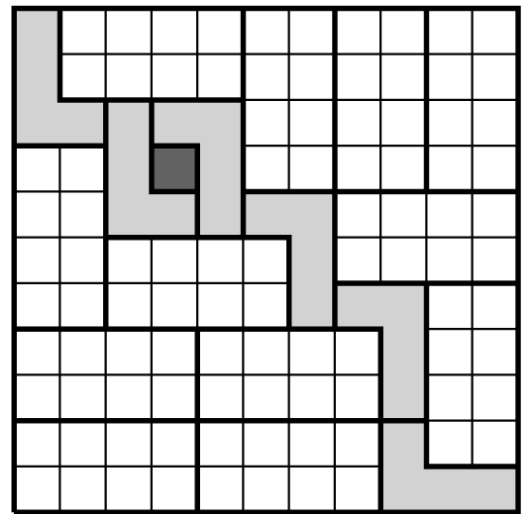

(a)

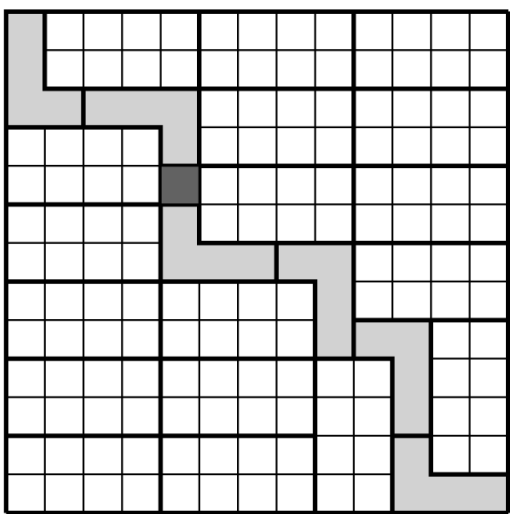

(b)

Figure 2. Tiling deficient squares. (a) $11 \times 11$ defcient square; (b) $13 \times 13$ defcient square.

$(4 m+3) \times(4 m+3)$. For all $m \geq 1$, all such positions give deficient squares that have tilings by $T_{4}^{+}$.

3. Most of the tiles (all but $n$ or $n+1$ ) in the tiling follow the rectangular pattern, that is, the tetrominoes are paired with each pair tiling a $2 \times 4$ rectangle.

4. (Existence of the crack) The $n$ or $n+1$ tiles in an irregular position together with the missing cell form a NW-SE diagonal crack. The crack starts in the upper left corner of the square and ends in the lower right corner.

5. (Location of the crack) The crack is located in a thin region symmetric about the diagonal, made out of a sequence of $3 \times 3$ squares that overlap over one of the corner cells.

6. The number of possible cracks (counted twice if they allow for two different tilings) in a fixed deficient square is $2^{2 m}$ if the square is $(4 m+1) \times(4 m+1)$ and $2^{2 m+1}$ if the square is $(4 m+3) \times(4 m+3)$.

7. The number of tilings of $a(4 m+1) \times(4 m+1), m \geq 1$, deficient square by $T_{4}^{+}$is equal to $N(m)=\sum_{k=1}^{2 m} 2^{k} N_{k}$, where $N_{k}$ is the number of tilings by do minoes and monomers of a $2 m \times 2 m$ square that has the diagonal covered by $k$ monomers and $2 m-k$ dominoes.

8. The number of tilings of a $(4 m+3) \times(4 m+3), m \geq 1$, deficient square by $\mathrm{T}_{4}^{+}$is $2 \mathrm{~N}(\mathrm{~m})$.

9. The countings are realized by surjective functions that take a tiling by $\mathrm{T}_{4}^{+}$ into a tiling by dominoes and monomers: for a tiling of the deficient square by $T_{4}^{+}$, eliminate the crack, replace the pairs of tiles that cover $2 \times 4$ rectangles by $2 \times 4$ rectangles, reassemble the remaining two region in a square and then do a $1 / 2$-homothety. If the image of a tiling by $T_{4}^{+}$has exactly $k$ monomers on the main diagonal, then the cardinality of the preimage of that image is $2^{k}$.

10. (Propagation of the crack) The crack and the tiling of $a(2 n+1) \times(2 n+1)$ square can be extended into a crack (tiling) of $a(2 n+5) \times(2 n+5)$ square.

Our results about the existence and the properties of tilings with cracks can be easily extended to more irregular regions, and even to regions with extra holes. The idea of the proof is to complete these regions to a full deficient square and 
then apply the results in Theorems 1 and 2. Some typical examples are shown in Figure 3. Tilings of deficient rectangles by $L$-tetrominoes, with all 8 symmetries allowed, are described in [4]. In contrast to what happens here, the obstructions to tilings in that case are mostly coloring invariants.

Other deficient regions of interest are quadrants, half strips or strips, bent strips, half planes or the plane, or a larger copy of the $L$-tetromino. All of these regions are considered in Golomb tiling hierarchy [5].

Theorem 3. a) No deficient first quadrant can be tiled by $T_{4}^{+}$. Every deficient second quadrant can be tiled by $T_{4}$. Moreover, no first quadrant missing a square of odd size can be tiled by $T_{4}^{+}$.

b) No deficient half strip can be tiled by $T_{4}^{+}$.

c) No deficient strip can be tiled by $T_{4}^{+}$.

d) No deficient bent strip can be tiled by $T_{4}^{+}$.

e) Every deficient half space can be tiled by $T_{4}$.

f) Every deficient plane can be tiled by $T_{4}$.

g) No deficient larger copy of an L-tetromino can be tiled by $\mathrm{T}_{4}^{+}$.

Combining the results in Theorems 1,2 and 3, we observe that $T_{4}$ and $T_{4}^{+}$ provide examples of tile sets that tile deficient squares of arbitrary large size, but do not tile a deficient first quadrant. This is in contrast with what happens for other tile sets. For example, it is known that the $T$-tetromino, with all symmetries allowed, does not tile deficient rectangles [6] [7], but it is easy to show that tiles deficient first quadrants. Theorems 2 and 3 show that the tiling hierarchy established by Golomb in [5] for tiling regions without holes fails for deficient regions and for tile sets allowing only for translations.

Some caution is needed in investigating a tiling hierarchy for deficient regions. For example, if the tile set consists of a single deficient rectangle then clearly it tiles a deficient rectangle (itself) but does not tile any infinite deficient region. The tile sets $T_{4}$ and $T_{4}^{+}$have the feature that the tiles do not have any holes. A finite tile set consisting of tiles without holes that tiles a deficient rectangle but does not tile any infinite region in the list above is shown Figure 4. We allow only for translations of the tiles. We observe that the tile sets $T_{4}$ and

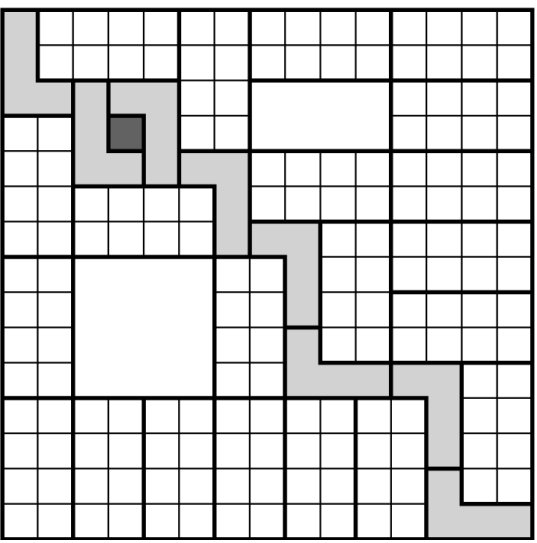

(a)

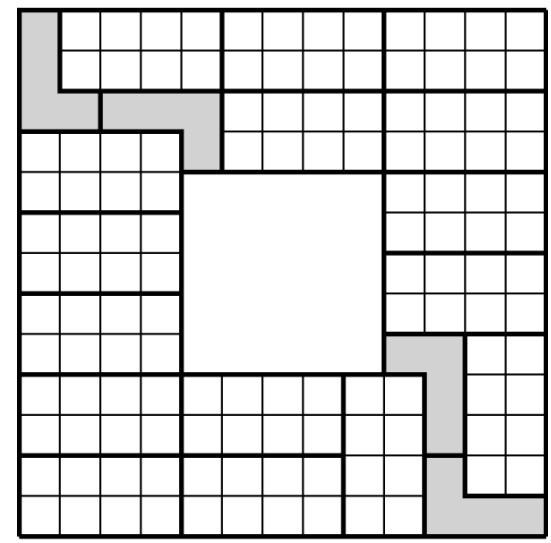

(b)

Figure 3. Tiling irregular regions. 

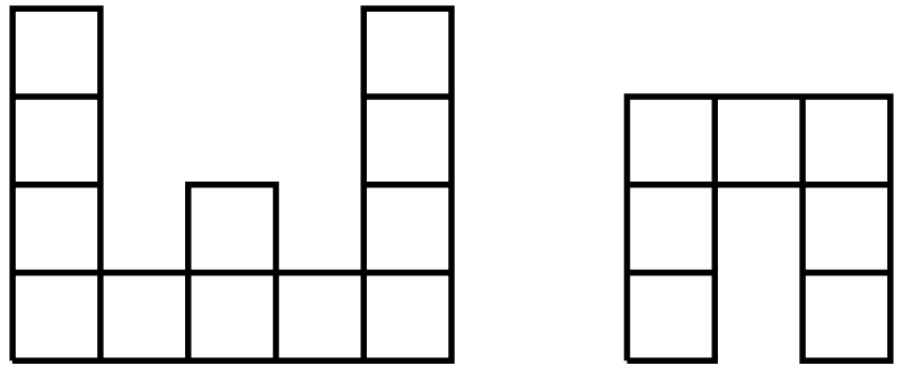

Figure 4. A tile set that tiles deficient rectangles but does not tile a deficient plane.

$T_{4}^{+}$have the extra feature that tile full rectangles. It is easy to see that a tile set that tiles a deficient rectangle and a rectangle, also tiles any deficient plane. One may consider the following problem.

Problem. Find a finite set of polyominoes that tiles rectangles, tiles deficient rectangles, but does not tile any deficient quadrant, deficient strip, deficient half strip, deficient bent strip or deficient half plane.

As we do not allow for all symmetries in our tile sets, it is also interesting to observe that the results in Theorem 3, b), c), d), e), g) are true for all orientations of the regions.

One may interpret tiling deficient regions in several ways. Given a region, we can consider all instances of deficient regions, almost all, a certain ratio, some or other variation. In Theorem 3 we consider all instances for both positive and negative results. In Theorems 1 and 2 only some instances of deficient squares are relevant, and the ratio of good cells versus the number of cells in the square converges to zero as the squares become larger and larger. For what the meaning of with probability $p$, may be and some results in this direction, one can consult [8] and the references cited there.

The results in this note can be generalized to tilings of deficient rectangles and other regions by the sets of ribbon $L n$-ominoes, $n$ even. These tile sets are introduced in [9]. Probably similar results may also be derived for more general tile sets appearing from dissection of rectangles that follow the rectangular pattern. These tile sets are described in [10] and [11].

\section{Proof of Theorem 1}

It follows from [1] that any tiling by $T_{4}$ of a region in the first quadrant bounded by the coordinate axes and a staircase of even origin and step 2 has to follow the rectangular pattern. For simplicity we will call such a region staircase. Inside any $(4 p+1) \times(4 q+1)$ or $(4 p+3) \times(4 q+3)$ rectangle we can fit two maximal regions as above. See Figure 5. Assume that the missing cell is not placed on the diagonal adjacent to one of the staircase regions or inside that region. This forces the appearance of the gray staircase in Figure 5 which ends in the cell ${ }^{*}$ that cannot be tiled. We conclude that the deficient rectangle has to be a square and the missing cell has to be placed on the diagonal.

Assume now that we have a deficient square. See Figure 6. It follows from [1] that all $2 \times 2$ marked squares are tiled following the rectangular pattern. We 


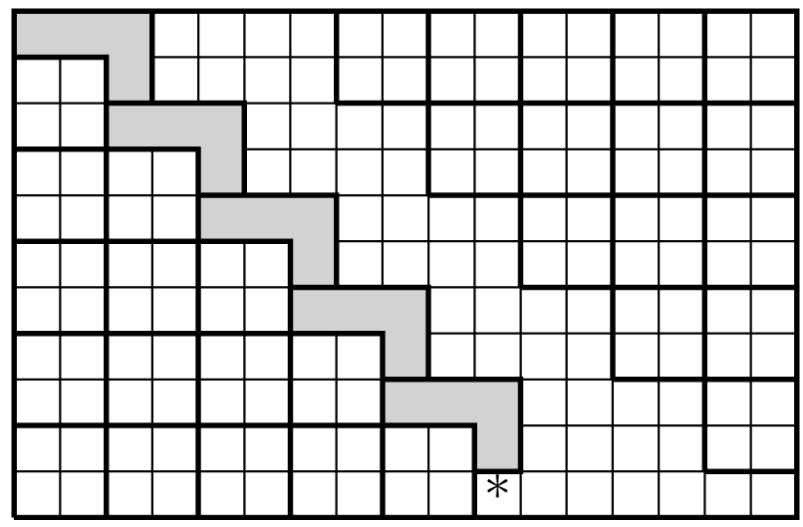

Figure 5. A general rectangle.

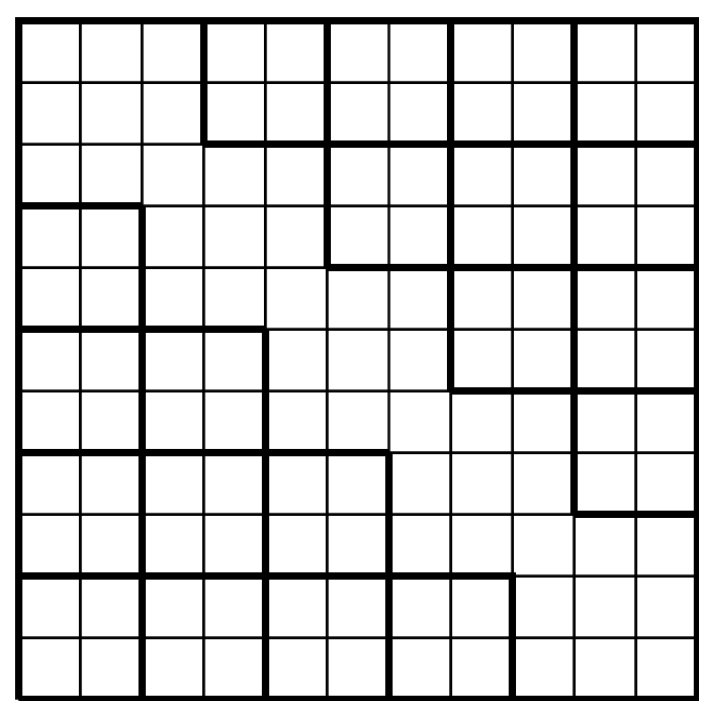

Figure 6. The central region.

study now the tiling of the remaining central region consisting of a sequence of $3 \times 3$ squares centered about the main diagonal. We observe first that some of the central region has to be covered by $2 \times 2$ squares that are parts of $2 \times 4$ rectangles originating in the region covered by the staircases. We will refer to them as 2-squares as well. Indeed, doing a chessboard coloring (say black, white) of the 2 -squares in the staircase region, we need to have the same number of black and white squares. This is due to the fact that any $2 \times 4$ rectangle is covered by a black and a white 2 -square. If the deficient square is

$(4 m+1) \times(4 m+1)$ then we have a deficiency of $m 2$-squares for each of the staircases and if the deficient square is $(4 m+3) \times(4 m+3)$ then we have a deficiency of $m 2$-squares for each of the staircases. In the first case this forces all $3 \times 3$ squares in the central region to contain a 2 -square and in the second case forces all but one of the $3 \times 3$ squares in the central region to contain a 2 -square. The 2 -squares are distributed evenly between the two staircases. The way in which we partition them in two equal parts, as we see below, can be arbitrary.

We study now the region left uncovered in the central region after the addi- 
tion of the 2 -squares. The possible tilings of a $3 \times 3$ square are shown in Figure 7. In cases a) through d) the cell labeled ${ }^{\star}$ has to be covered by the missing cell in the deficient square or by a cell that is part of a tile in $T_{4}$ originating in a different $3 \times 3$ square. In cases e) and f) the central cell in $3 \times 3$ square has to be the missing cell. If the deficient square is of type $(4 m+1) \times(4 m+1)$ then the $3 \times 3$ squares are covered by the cases a) through $d$ ). The missing cell has to be in an odd position on the main diagonal covering the corner of a $3 \times 3$ square. If the deficient square is of type $(4 m+3) \times(4 m+3)$ then all but one of the $3 \times 3$ squares are covered by the cases a) through $d$ ) and one of them is covered by the case e) or $\mathrm{f}$ ). The missing cell has to be in an even position on the main diagonal covering the center of a $3 \times 3$ square. It is easy to see that once the 2squares and the missing cell are places in the central region, the rest of the crack can be tiled by $T_{4}$ in a unique way for $(4 m+1) \times(4 m+1)$ squares and in two ways for $(4 m+3) \times(4 m+3)$ squares.

We show now that after the addition of the 2 -squares to the staircase the resulting region can be tiled by $2 \times 4$ rectangles. This is obvious if the number of rows in the staircase is 1 or 2 . If the number of rows is larger, we do induction on the number of rows. The induction step is illustrated in Figure 8 and consists in removing $2 \times 4$ rectangles containing the additional 2 -squares. We start removing $2 \times 4$ rectangles from the top of the staircase. Each removal deletes also a 2 -square on the largest diagonal of the staircase. In order to avoid ambiguity, we want the 2-squares on the diagonal to be eliminated in order, from the top to the bottom. What is left is a smaller staircase with the additional 2 -squares added which can be tiled by $2 \times 4$ rectangles due to the induction hypothesis.

To finish the proof of the counting results we need to show that any tiling by dominoes of a $2 m \times 2 m$ square or of a $(2 m+1) \times(2 m+1)$ deficient square with the missing cell on the main diagonal can be divided in tilings of complementary staircases regions with extra squares added. Observe that any of the $2 m$ available cells on the diagonal is covered by a different domino. To obtain the splitting, divide the set of dominoes that cover the cells on the diagonal in two equal parts and assign them to the lower, respectively upper, maximal staircase in the original $2 m \times 2 m$ or $(2 m+1) \times(2 m+1)$ square. This argument also allows to count the number of cracks. The crack is uniquely determined by the partition of extra $2 \times 2$ squares in two equal parts between the upper and lower staircases. Independent of the size of the deficient square, this partition can be done in $C_{2 m}^{m}$ ways.

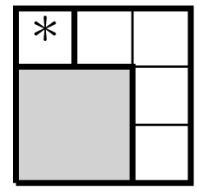

(a)

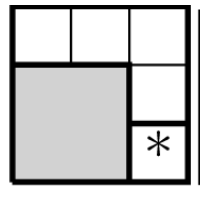

(b)

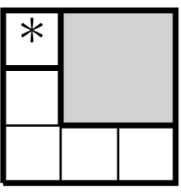

(c)

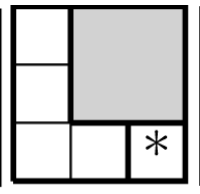

(d)

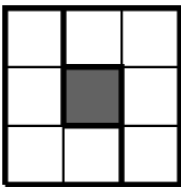

(e)

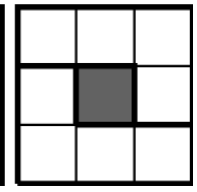

(f)

Figure 7. Tilings of a $3 \times 3$ square. 
The propagation of the crack is illustrated in Figure 9 and it is self-explanatory.

\section{Proof of Theorem 2}

The proof of Theorem 2 is similar to that of Theorem 1. Figure 7 shows that the appearance of the extra $2 \times 2$ square inside the $3 \times 3$ squares is forced and the only possible coverings of a $3 \times 3$ square are shown in that figure. The differences in counting appear due to the fact that the extra $2 \times 2$ squares that are added in the central region do not need to be divided in two equal parts, but rather can be divided arbitrarily. If the extra $2 \times 2$ squares are placed, then the region covered by the crack is well defined. As a $2 \times 2$ square can be placed in only two positions, the number of cracks is $2^{2 m}$ for a $(4 m+1) \times(4 m+1)$ square and $2 \times 2^{2 m}$ for a $(4 m+3) \times(4 m+3)$ square. The extra factor of 2 appears due to the fact that in the last case the region supporting the crack can be tiled in two ways. See Figure 7(e), Figure 7(f). Same arguments justify the extra factors of $2^{k}$ and 2 appearing in the statements 7 and 8 in Theorem 2.

\section{Proof of Theorem 3}

a) In order to prove first part in a) we use a technique introduced in [1] for
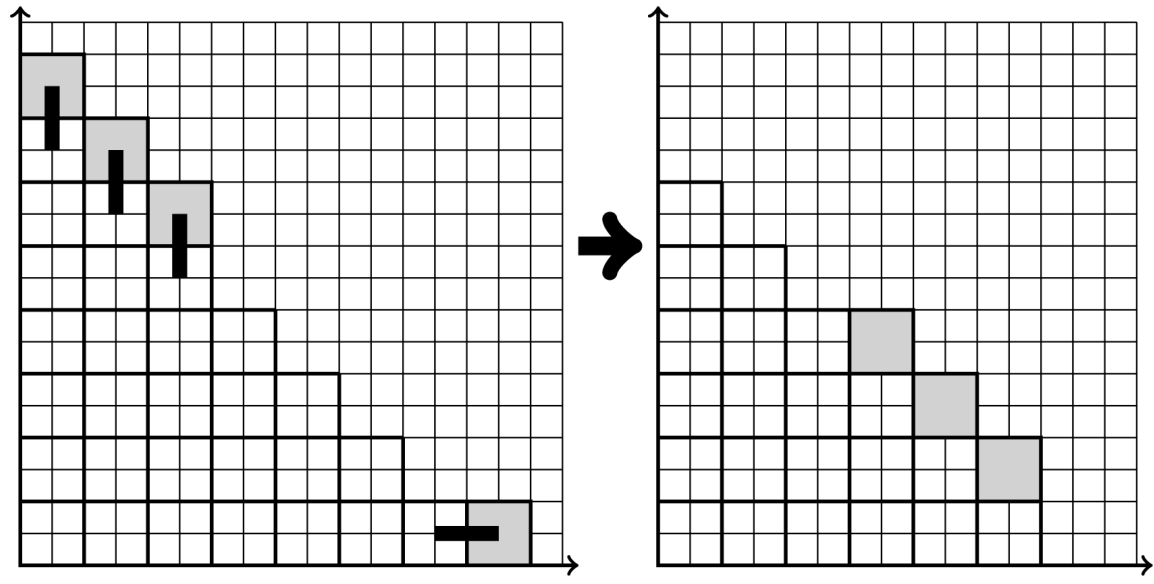

Figure 8. The induction step.

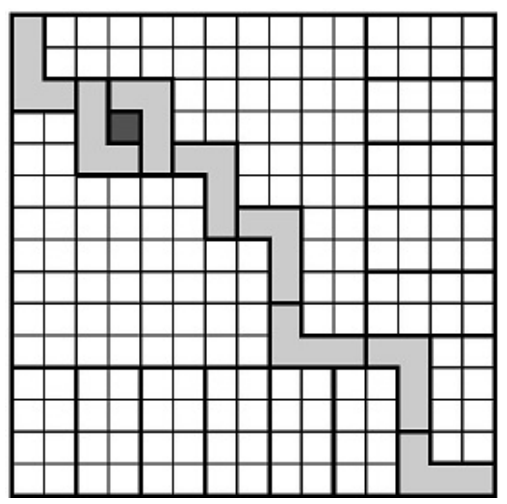

Figure 9. Propagation of the crack. 
$T_{4}$. Any line parallel to $y=-\chi$ that intersects a tile from $T_{4}$ across diagonals of cells, cuts either a cell or two cells from each tile. This leads to a $(1,2,1)$ invariant, which is available even if an extra $2 \times 2$ tile is added to the tile set. We label the diagonal in the first quadrant by integers, starting from the corner of the first quadrant. Using the invariant it is shown in [1] that in any tiling of a (non deficient) first quadrant even diagonals parallel to $y=-x$ are tiled by the blocks of two cells belonging to the same tile and the odd diagonals are tiled by single cells belonging to distinct tiles. In a tiling of a deficient first quadrant, this pattern has to hold till we reach the diagonal containing the missing cell.

Assume that the missing cell is placed on an even diagonal $D_{2 n}$ of length $2 n$. As the tiling of $D_{2 n-1}$ is done by single cells belonging to distinct tiles, this forces $D_{2 n}$ to be tiled by the blocks of two cells belonging to the same tile, so taking out a cell from $D_{2 n}$ leads to a contradiction.

Assume now that the missing cell is placed on an odd diagonal $D_{2 n+1}$ of length $2 n+1$. Then the diagonal $D_{2 n}$ is covered by $n$ tiles, each tile covering two cells from $D_{2 n}$. These tiles also cover $n$ cells from $D_{2 n+1}$. After taking out the missing cell, the rest of $n$ cells of $D_{2 n+1}$ are covered by single cells from tiles that also cover $2 n$ cells from the diagonal $D_{2 n+2}$ and $n$ cells from the diagonal $D_{2 n+3}$. The two cells left uncovered on $D_{2 n+2}$ are covered by single cells from distinct tiles, which also cover 4 cells from $D_{2 n+3}$ and 2 cells from $D_{2 n+4}$. So, it follows that the number of new tiles that cover the rest of the diagonals is forced. By induction, one can easily show that for any $k \geq 2$ and even, the number of new tiles that appear in its cover is $k$. Once $k>2 n+1$, this forces $D_{2 n+k+1}$ to have at least $2 k$ cells, in contradiction to $2 k>2 n+k+1$. This finishes the proof for the first part of a).

Several tilings of deficient second quadrants are shown in Figure 10. The half infinite strips in the figures can be tiled by $2 \times 4$ rectangles. Tilings for the rest of the cases can be obtained from these via a symmetry about the line $y=-x$ or a translation that leaves in the second quadrant a region that can be tiled by $2 \times 4$ rectangles. This proves second part of a).

Third part of a) follows combining first part of a) with Theorem 2 .

b) If a deficient half strip is tiled by $T_{4}$, then a deficient first quadrant or a deficient third quadrant is tileble by $T_{4}$. But this is in contradiction to a).

c) Due to the $(1,2,1)$ invariant mentioned above, in any tiling of a deficient infinite strip, the diagonals inside the strip parallel with the line $y=-x$ are covered either by blocks of two cells of by single cells belonging to individual tiles.

The proof is similar to that in [1], showing that a double infinite strip of odd width cannot be tiled by $T_{4}$, but here we have more cases to consider. Clearly a deficient strip of width 1 or 2 cannot be tiled. We show first a stronger result that implies impossibility of tiling a deficient strip of width 3 or 4 . If a tiling is possible, the infinite sequences $(\ldots, 3,3,3,3,2,3,3,3, \ldots),(\ldots, 4,4,4,4,3,4,4,4, \ldots)$ are linear combinations with positive coefficients of translates of the block $(1,2,1)$.

Consider the first infinite sequence and look at the diagonal containing the missing cell, say $D_{0}$. Label the rest of the diagonals from left to right. If $D_{0}$ is 


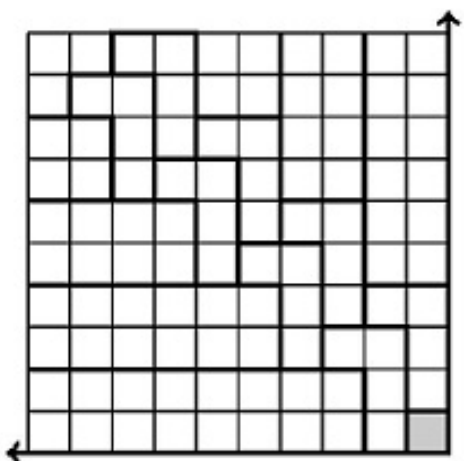

(a)

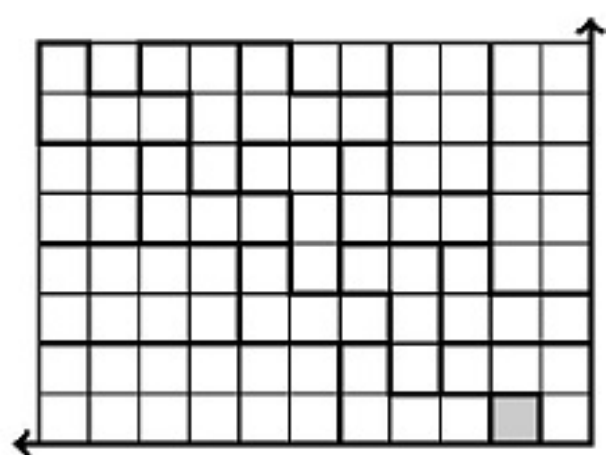

(b)

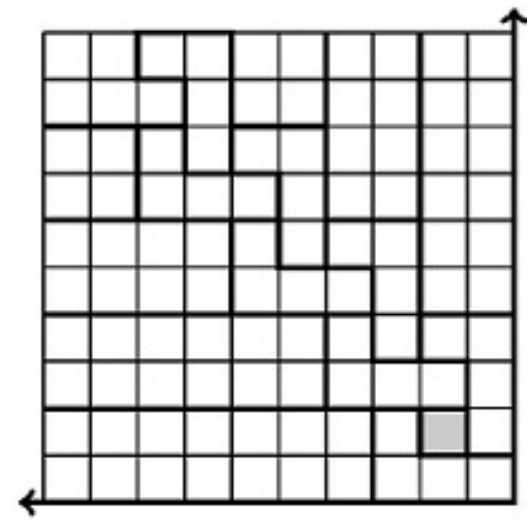

(c)

Figure 10. Tilings of a deficient second quadrant.

tiled by a block of two cells belonging to a single tile, then the diagonal $D_{2}$ cannot be tiled. If $D_{0}$ is tiled by two single cells belonging to different tiles, then $D_{4}$ cannot be tiled.

Consider now the second infinite sequence and look at the diagonal $D_{0}$ that contains the missing cell. If $D_{0}$ is tiled by single cells belonging to different tiles, then either $D_{1}$ or $D_{-1}$ is covered by a block of two cells and two single cell, which make $D_{2}$ or $D_{-2}$ impossible to tile. If $D_{0}$ is tiled by a block of two cells and a single cell, then one of $D_{1}$ or $D_{-1}$ is covered by a block of two cells and two single cells, and the other is tiled by four single cells. This forces one of $D_{2}$ or $D_{-2}$ to be impossible to tile.

For the induction step, the case of width $k, k$ odd, notice that for any diagonal there is at least a block of two cells from the same tile part of its cover. Otherwise one of the adjacent diagonals is forced to have more than $k$ cells. Then we may subtract an infinite sequence $(\ldots, 4,4,4, \ldots)$ from the invariant associated to the whole strip and reduce the problem to a deficient strip of width $k-4$.

For the induction step, the case of width $k, k$ even, the above argument works if we show that the existence of a diagonal covered only by single cells from distinct tiles gives a contradiction. Assume that the diagonal containing the missing cell, $D_{0}$, is covered only by single cells. Then either $D_{1}$ (say) (or $D_{-1}$ ) is covered by $k-1$ blocks of two cells and two single cells. This forces $D_{2}$ to 
be covered by two blocks of two cells and $2 k-4$ single cells, $k-1$ of which belong to tiles covering the previous diagonals. By induction, this shows that the diagonal $D_{2 n}$ is covered by $2 n$ blocks of two cells, which leads to a contradiction if $2 n>k$. If a diagonal different from one with the missing cell is covered only by single cells, then the adjacent diagonals have to be completely covered by blocks of two cells or by single cell belonging. This determines a pattern that leads to a contradiction as we reach the diagonal containing the missing cell.

d) The proof of c) and a) use only the $(1,2,1)$ invariant, so the argument is also available for a bent strip.

e) This easily follows from a).

f) This easily follows from a).

g) Obvious due to a counting argument.

\section{Acknowledgements}

V. Nitica was partially supported by Simons Foundation Grant 208729.

\section{References}

[1] Chao, M., Levenstein, D., Nitica, V. and Sharp, R. (2013) A Coloring Invariant for Ribbon L-Tetrominoes. Discrete Mathematics, 313, 611-621. https://doi.org/10.1016/j.disc.2012.12.007

[2] Nitica, V. (2003) Rep-Tiles Revisited, in the Volume MASS Selecta: Teaching and Learning Advanced Undergraduate Mathematics. American Mathematical Society.

[3] Kasteleyn, P.M. (1961) The Statistics of Dimers on a Lattice. Physica, 27, 1209-1225. https://doi.org/10.1016/0031-8914(61)90063-5

[4] Nitica, V. (2004-2005) Tiling a Deficient Rectangle by L-Tetrominoes. Journal of Recreational Mathematics, 33, 259-271.

[5] Golomb, S.W. (1994) Polyominoes, Puzzles, Patterns, Problems, and Packings. 2nd Edition, Princeton University Press.

[6] Hochberg, R. (2015) The Gap Number of the T-Tetromino. Discrete Mathematics, 338, 130-138. https://doi.org/10.1016/j.disc.2014.09.001

[7] Zhan, S. (2012) Tiling a Deficient Rectangle with t-Tetrominoes. https://www.math.psu.edu/mass/reu/2012/report/Tiling Deficient Rectangles with T-Tetrominoes.pdf

[8] Junius, P. and Nitica, V. (2017) Tiling Rectangles with Gaps by Ribbon Right Trominoes. Open Journal of Discrete Mathematics, 7, 87-102. https://doi.org/10.4236/ojdm.2017.72010

[9] Nitica, V. (2015) Every Tiling of the First Quadrant by Ribbon $L$ n-Ominoes Follows the Rectangular Pattern. Open Journal of Discrete Mathematics, 5, 11-25. https://doi.org/10.4236/ojdm.2015.52002

[10] Calderon, A., Fairchild, S., Nitica, V. and Simon, S. (2016) Tilings of Quadrants by L-Ominoes and Notched Rectangles. Topics in Recreational Mathematics, 7, 39-75.

[11] Nitica, V. (2016) On Tilings of Quadrants and Rectangles and Rectangular Pattern. Open Journal of Discrete Mathematics, 6, 252-271.

https://doi.org/10.4236/ojdm.2016.64028 
Submit or recommend next manuscript to SCIRP and we will provide best service for you:

Accepting pre-submission inquiries through Email, Facebook, LinkedIn, Twitter, etc. A wide selection of journals (inclusive of 9 subjects, more than 200 journals)

Providing 24-hour high-quality service

User-friendly online submission system

Fair and swift peer-review system

Efficient typesetting and proofreading procedure

Display of the result of downloads and visits, as well as the number of cited articles Maximum dissemination of your research work

Submit your manuscript at: http://papersubmission.scirp.org/

Or contact ojdm@scirp.org 